\title{
Les dictionnaires de synonymes : une typologie évoluant avec le temps
}

\author{
Ferrara, Alice \\ Université de Cergy-Pontoise, \\ Laboratoire LDI (CNRS UMR 7187) \\ alice.ferrara@gmail.com
}

\section{Introduction}

Depuis 1718, la lexicographie française bénéficie d'un type de dictionnaire spécialisé de la langue : le dictionnaire de synonymes. Cette catégorie de dictionnaire a connu une grande évolution depuis sa naissance. En effet, le dictionnaire de synonymes actuel n'a vu le jour qu'au cours du $\mathrm{XX}^{\mathrm{e}}$ siècle. Préalablement, il ne comportait pas les mêmes renseignements et n'avait donc pas la même fonction. À travers le temps, il a existé, parfois coexisté, quatre types de dictionnaires chacun révelant la vision des synonymistes sur leur objet d'étude. Ces différents types de dictionnaires ont nécessairement changé les habitudes de recherche de termes synonymes pour le lecteur. Ainsi, avec l'évolution du genre, qui a suivi le temps, les informations apportées par les dictionnaires de synonymes ne sont plus les mêmes.

Afin de mener à bien notre étude, il convient de commencer par exposer les quatre différents types de dictionnaires de synonymes, puis d'observer les particularités de chacun de ces genres et enfin, d'analyser ce qu'apportent à l'ouvrage les différentes informations fournies. Afin de limiter l'analyse, nous avons choisi d'établir un corpus restreint se composant d'ouvrages particuliers. Les dictionnaires mentionnés sont donc représentatifs de la catégorie auxquels ils appartiennent.

\section{Les dictionnaires de synonymes}

\subsection{Qu'est ce qu'un dictionnaire de synonymes ?}

Tous les dictionnaires ne sont pas du même type. Il en existe un grand nombre, qui ne tiennent pas compte des mêmes critères. En dehors des traditionnels dictionnaires généraux, il existe des dictionnaires spécialisés. Dans un dictionnaire spécialisé,

Les mots ne [sont] examinés que sous un angle particulier, formel ou sémantique, et donc choisis en fonction d'une caractéristique linguistique déterminée au préalable. Pruvost (2006: 136)

Le dictionnaire de synonymes rentre donc dans cette catégorie de dictionnaires spécialisés de la langue car il renferme des termes choisis pour leurs caractéristiques sémantiques. Il ne possède pas la même nomenclature qu'un dictionnaire général. Tous les mots ne sont pas consignés, seuls ceux qui ont une relation synonymique avec un autre. Un dictionnaire de synonymes est un ouvrage qui consigne des termes que l'on pourrait confondre, qui désignent la même chose, ou qui, selon les contextes peuvent être interchangés. Cependant dans la préface de son dictionnaire, Boussinot souligne une contradiction :

Tout dictionnaire de synonymes est fondé sur un paradoxe. Aucun mot de la langue française n'est en effet rigoureusement synonyme d'un autre : chacun recèle une nuance qui lui est propre, qui le différencie et qui, d'ailleurs, justifie son existence. [...] On peut donc dire qu'il n'y a pas de synonymes. Boussinot $(2008: 7)$

Si les synonymes n'existent pas, les dictionnaires de synonymes ne devraient pas exister. Mais justement, au $\mathrm{XVIII}^{\mathrm{e}}$ siècle, on trouvait l'intérêt des dictionnaires de synonymes dans le fait que les auteurs 
cherchaient à expliquer les différences qui séparaient les mots, les détails qui faisaient qu'on ne devait pas les confondre. Aujourd'hui, il y a une évolution dans l'utilité de ce type de dictionnaire et la démarche des synonymistes. Bien que conscients que les synonymes n'existent pas réellement, les auteurs de dictionnaires de synonymes, n'hésitent pas à offrir à leurs lecteurs des listes de termes considérés comme voisins, mais sans leur donner les moyens, à travers une définition ou des exemples, de savoir ce qui les distingue.

\subsection{Naissance d'un genre}

En 1718 Girard publie Justesse de la langue françoise, dont le but est de donner, comme l'indique le sous-titre, « les différentes significations des mots qui passent pour synonimes. »

$\mathrm{Au} \mathrm{XVIII}^{\mathrm{e}}$ siècle, la vision de la synonymie évolue car préalablement, passaient pour synonymes les termes qui en réalité étaient analogues. En effet, avant ce siècle, le mot « synonyme » s'appliquait dans une relation d'analogie comme en témoigne la citation d'Aristote :

Sont dits synonymes les choses dont le nom est commun et dont l'énoncé de l'essence conforme au nom est le même, par exemple sont dits « animal » l'homme et le bœuf. En effet, l'homme et le bœuf sont appelé « animal » par un nom commun et l'énoncé de son essence est le même, car si l'on donne l'énoncé de chacun, c'est-à-dire ce qu'est pour chacun d'eux le fait d'être animal, on donnera le même énoncé. Aristote, Catégories, cité dans Annick Stevens (2000:63)

C'est au XVIII ${ }^{\text {e }}$ siècle, au moment de l'apogée des dictionnaires de synonymes distinctifs, que la définition moderne de la synonymie s'établit. À partir de ce siècle, on entend par « synonymes »: mots de même sens. Cependant, au moment même où l'on affirme que les synonymes sont des mots de même sens, on affirme également qu'étant donné que c'est rare, les mots n'ont pas forcément le même sens, mais une idée commune. Ce sont des synonymistes tels que Girard ou Roubaud qui ont établi la synonymie telle que nous la connaissons.

Avant la parution du dictionnaire de Girard, il existait déjà des ouvrages sur la synonymie comme Synonimes de Gérard de Vivre (1569), qui est un ouvrage bilingue français-allemand, ou Synonimes et épithètes françoises d'Antoine de Montmeran (1645), mais l'auteur retient plus les épithètes que les synonymes. C'est pourquoi l'ouvrage de Girard est véritablement le premier du genre, qui fera, par la suite, beaucoup d'émules.

Lorsqu'il compose son dictionnaire de synonymes, Girard veut faire un ouvrage afin

Qu'on apprenne à ne les [synonymes] employer qu'à propos, à ne les points confondre, \& à les mettre précisément à leurs places.

Girard (1718: xvj)

Le but premier d'un dictionnaire de synonymes était pédagogique, leurs auteurs ont voulu enseigner le bon emploi des termes.

Soulignons que, à la naissance du genre, le fait de vouloir créer un dictionnaire de synonymes pour différencier les termes n'est pas une exclusivité française. En effet, en Espagne, les synonymistes ont la même motivation :

En los diccionarios de sinónimos sí se han podido ver planteamientos totalmente explícitos, sobre todo en la lexicografía de los siglos XVIII y XIX (González Pérez, 1994), ya que se elaboraban obras para marcar las diferencias entre los vocablos, lo que conlleva la negación de la sinonimia absoluta, si bien en los últimos años, y salvando algunas excepciones, predominan los repertorios acumulativos, muy denostados por su escaso valor didáctico.

Garcia Platero (2008 : 352) 


\subsection{De véritables traités de synonymie}

Les dictionnaires de synonymes du XVIII ${ }^{\mathrm{e}}$ siècle, bien plus que de simples dictionnaires, sont de véritables traités de synonymie. En effet, ces ouvrages sont précédés de préfaces et introductions dans lesquelles les auteurs apportent leur vision très personnelle sur la manière de traiter des synonymes, et de les consigner dans des ouvrages spécialisés. C'est pourquoi il est fort utile d'analyser les préfaces des dictionnaires du corpus avant de se pencher sur le contenu des articles pour comprendre quelle était la vision des synonymistes des siècles précédents.

Dans leurs préfaces respectives, Roubaud et Girard s'accordent à dire que la clarté d'une langue est très importante. Or cette clarté passe par la connaissance exacte des termes, la valeur des mots. De plus, les deux synonymistes affirment que la richesse d'une langue ne tient pas au nombre de termes qu'elle possède, mais aux mots qui expriment les différences car la surcharge de mots appauvrit l'idée exprimée.

Dans sa préface Laveaux cherche à expliquer l'importance du titre qu'il donne à son dictionnaire, Dictionnaire synonymique de la langue française (1826). Pour lui, les synonymes n'existent pas, il faut donc parler de dictionnaire « synonymique », c'est-à-dire qui a un rapport avec la synonymie, mais n'en est pas.

De manière générale, dans les préfaces des dictionnaires du XVIII ${ }^{\mathrm{e}}$ siècle, les auteurs cherchent en priorité à montrer l'importance d'un tel dictionnaire. C'est-à-dire qu'ils font un dictionnaire de synonymes car certains mots voisins ont des nuances trop subtiles; ainsi certains locuteurs peuvent ne pas être aptes à faire la différence entre les deux termes.

De plus, à partir du moment où Roubaud a introduit l'étymologie dans son dictionnaire, les synonymistes ont tous donné leurs avis sur la question dans leurs préfaces. Ainsi nous apprenons notamment que pour Guizot le recours à l'étymologie semble primordial lors de l'étude des synonymes :

\section{L'étymologie apprend aussi à connaitre le sens primitif, et par conséquent le sens propre des termes. [...] Elle est le seul flambeau à la lumière duquel on puisse étudier les langues, et sur-tout les rapports de synonymie qui existent entre les mots. \\ Guizot (1809:vj)}

Et enfin, des aspects grammaticaux peuvent faire l'objet d'études dans les préfaces. Ainsi, Guizot consacre la fin de sa préface à l'explication morphologique des terminaisons des mots. Contrairement à Roubaud qui le fait tout au long de ses articles, Guizot a choisi de ne pas le faire au sein du dictionnaire pour chaque terme, mais de façon globale en introduction.

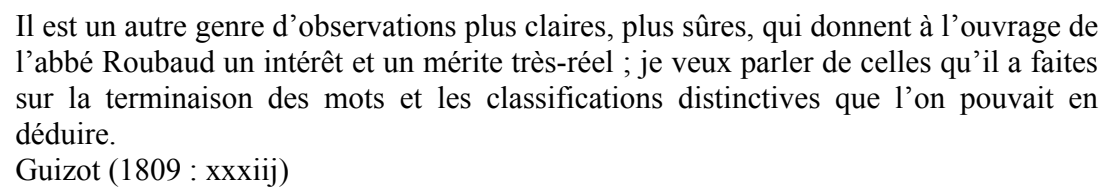

Les préfaces et introductions des dictionnaires de synonymes du XVIII ${ }^{\mathrm{e}}$ siècle sont donc très riches et permettent de consulter le dictionnaire tout en ayant connaissance de l'avis du synonymiste sur la synonymie, la manière de la traiter.

\section{Les quatre types de dictionnaires de synonymes}

\subsection{Les distinctifs}

\subsubsection{Des dictionnaires de comparaison}

Les dictionnaires de synonymes distinctifs se composent de définitions, de précisions et d'exemples qui justifient les propos de l'auteur. Le synonymiste analyse les différences existantes entre les termes considérés comme synonymes. Dans ce type de dictionnaire, ne sont pas présents tous les synonymes 
quel que soit le contexte. Le synonymiste propose un sens dans lequel les mots se rapprochent, il montre les différences, mais ne définit le terme que dans un sens possible. C'est donc très restrictif. Le désavantage de ce type de dictionnaire est qu'on ne peut pas trouver de synonymes dans des contextes différents. Ce type de dictionnaire ne se consulte pas d'après un mot-vedette, mais deux ou trois. En effet, le synonymiste interroge les ressemblances et différences de plusieurs termes. Ce sont donc des dictionnaires à entrées multiples ${ }^{1}$.

\subsubsection{Girard et Roubaud les deux fondateurs du genre}

Les deux ouvrages qui ont marqué ce genre, sont ceux de Girard ${ }^{2}$ et Roubaud ${ }^{3}$. L'importance de Girard réside dans le fait qu'il est le premier auteur de dictionnaire de synonymes monolingue français. Voyant que certains termes peuvent être confondus, et surtout ne pas être compris à leur juste valeur, Girard a décidé de composer son ouvrage pour éclairer le public. Il publie donc en 1718, La Justesse de la langue françoise, qui sera réédité en 1736 et 1740 . C'est un dictionnaire de synonymes distinctif qui comporte des entrées multiples où les termes sont mis en relation afin de déterminer ce qui les différencie. Ainsi, les trois premières entrées sont : "Accés, Abord, Approche ", "Action, Acte », et, " Adresse, Souplesse, Finesse, Ruse ». Nous constatons alors qu'il peut y avoir dans les dictionnaires de synonymes distinctifs, des entrées multiples avec deux, trois ou même quatre termes mis en comparaison. En réalité, dans les dictionnaires distinctifs le nombre de termes peut aller jusqu'à 14 .

Quant à Roubaud, il est fondamental dans l'histoire des dictionnaires de synonymes car il est le premier, et le seul à sa façon, à donner comme critère de différenciation des termes dits "synonymes ", l'étymologie dans les Nouveaux synonimes français en 1785. C'est tout à fait novateur. Ainsi il est le seul à se différencier de Girard, pas dans la forme qu'il donne à son dictionnaire, puisque c'est un distinctif à entrées multiples, mais la différence vient de la manière de différencier les termes. Il n'est pas en total désaccord avec son prédécesseur, mais désire aller plus loin. Il explique dans sa préface qu'il a choisi de tirer de l'étymologie la valeur des synonymes.

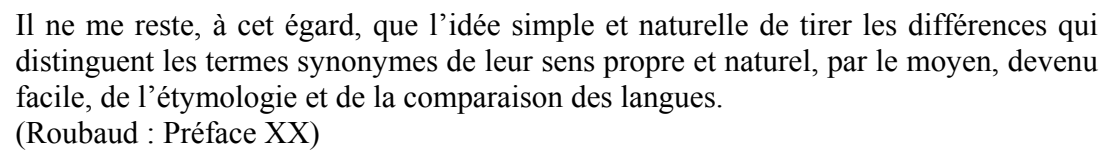

Il usera, et même abusera de l'étymologie dans la totalité de son ouvrage. Dès la première entrée "Abaisser, Rabaisser, Ravaler, Avilir, Humilier », il est indiqué que "Abaisser vient de bas, mot celtique ». Il prend donc appui sur l'étymologie pour trouver en quoi deux mots qui peuvent ne pas paraître synonymes à première vue le sont.

Le dictionnaire de Roubaud est très complet, il renseigne sur l'histoire des mots, la grammaire, et offre de nombreuses citations.

\subsection{Les compilations}

\subsubsection{Un travail d'emprunt}

Quand les dictionnaires de synonymes se sont multipliés, à peine un siècle après la naissance du genre, apparait un genre annexe, la compilation. Le but des auteurs de ces ouvrages de compilation était de garder ce qu'ils jugeaient être le meilleur des dictionnaires de synonymes les précédant. Des auteurs comme François Guizot, Benoît Morin ou encore Jean-Pons Victor de Levizac vont composer des dictionnaires de synonymes avec des articles de dictionnaires de synonymes déjà publiés. Pour cela, ils vont garder ce qui les intéresse dans les articles de leurs prédécesseurs, retrancher tout ce qu'ils trouvent être superflu et ajouter parfois, plus ou moins selon les auteurs, des articles qu'ils vont écrire eux-mêmes. Ainsi, Guizot sera l'auteur de 122 articles de son dictionnaire qui en contient 1341. Tandis que Levizac n'en compose que très peu, quatre au total. D'ailleurs dans sa préface, il précise : « il n’y a presque rien 
de moi dans cet ouvrage ». De plus, certains articles dans ces dictionnaires sont composés par différents auteurs puisque les compilateurs n'hésitent pas à prendre quelques phrases d'un auteur et quelques phrases d'un autre afin de composer un article complet. Bien que ce soit un travail basé sur l'emprunt, les compilateurs de dictionnaires de synonymes sont des synonymistes à part entière puisqu'ils établissent des choix concernant les mots-vedettes, les définitions, les étymologies, etc ${ }^{4}$.

\subsubsection{Les principales compilations : Guizot et Sardou}

L'ouvrage de référence pour les dictionnaires de compilation est celui de Guizot. En effet, le politiciensynonymiste en composant le Nouveau Dictionnaire universel des synonymes de la langue française reste dans la lignée de Roubaud en affirmant dans sa préface que l'étymologie est très importante lorsqu'il s'agit de synonymie. Cependant, il est beaucoup plus modéré que son aîné puisqu'il pense qu'il faut regarder aussi dans d'autres directions. Guizot publie son ouvrage en 1809. C'est une compilation des dictionnaires qui l'ont précédé, ainsi que 122 articles qu'il a composés lui-même. Le lecteur sait toujours, lorsqu'il lit un article, qui en est l'auteur, puisque des initiales de indiquent la personne qui a écrit l'article.

$\mathrm{Au}$ cours du XIX $\mathrm{e}^{\mathrm{e}}$ siècle, très peu de nouveaux dictionnaires d'un seul auteur voient le jour, ce sont principalement des recompositions, dont l'ouvrage de Sardou. Ce dernier est beaucoup plus critique que Guizot et réécrit plus d'articles. Son ouvrage est destiné aux élèves en priorité, comme il l'indique dans la préface "Un Nouveau Dictionnaire des synonymes français, spécialement destiné à l'enseignement public. ${ }^{5} »$ Il le compose principalement pour rectifier ce qu'il juge être mauvais dans les autres ouvrages de ce genre.

Parmi le petit nombre de ceux qui existent, aucun ne m'a paru satisfaisant, ni au point de vue de la philologie, ni à celui de l'éducation et de l'enseignement. Dans les uns, j'ai trouvé un assez bon nombre d'inexactitudes ; dans les autres, une foule de fautes typographiques qui dénaturent le sens et la pensée.

Sardou (1866: 4)

Pour son époque, le dictionnaire de Sardou couvre un grand nombre de mots, ce qui n'est pas habituel ${ }^{6}$. Certes son ouvrage est entièrement distinctif, mais il s'étend beaucoup moins sur les raisons qui distinguent les mots, pour se concentrer sur les distinctions en elles-mêmes. C'est-à-dire qu'il indique en les insérant dans son dictionnaire, que les termes sont synonymes et donc à distinguer. Cependant, il ne s'étend pas grâce à une longue distinction faite de définitions et illustrée d'exemples comme le font ses contemporains. Il reste assez concis. Il prend en compte les termes dans plus de contextes différents que ses prédécesseurs. Ainsi le terme action se trouve dans les entrées : «Acte, Action », et « Bonnes actions, Bonnes œuvres ». En effet, à la fin du $\mathrm{XIX}^{\mathrm{e}}$ siècle, le cheminement vers les dictionnaires cumulatifs commence. Dans un premier temps, dans le nombre d'entrées uniquement, car la distinction caractéristique des dictionnaires distinctifs se prolonge dans les définitions. Cependant, il commence également à y avoir des renvois comme : "Adage voir Proverbe ». Et une bonne partie des termes se trouvant dans les dictionnaires généraux se trouve également ici avec un synonyme l'accompagnant, ainsi qu'une explication très succinte de la principale différence qui les sépare.

\subsection{Les semi-distinctifs}

\subsubsection{Un genre hybride}

Entre les deux types de dictionnaire de synonymes que sont les cumulatifs et les distinctifs, il existe une sous-classe, qui n'est pas véritablement un genre à lui-seul, mais plutôt une variation. C'est le dictionnaire « semi-distinctif». Il s'approche du dictionnaire distinctif dans la démarche, mais est loin de comporter les distinctions suffisantes. C'est un dictionnaire qui se présente sous forme de liste. Le Nouveau Dictionnaire des Synonymes ${ }^{7}$ (1977) est un dictionnaire semi-distinctif. Ce n'est pas complètement un dictionnaire cumulatif car dans cet ouvrage, les termes sont distingués par des contextes 
et exemples. Mais ce n'est pas un dictionnaire distinctif comme celui de Girard car il n'y a pas de définitions afin d'expliquer les différences qu'il y a entre les termes. Les auteurs de ce dictionnaire se sont plus occupés du contexte variable que des sens possibles. Ce qui le rapproche des dictionnaires distinctifs et fait qu'il est à la jonction des deux genres principaux, c'est qu'il est illustré d'exemples ce qui est totalement exclu des dictionnaires cumulatifs. Le dictionnaire semi-distinctif permet aisément de montrer au lecteur les différents registres de langue ${ }^{8}$.

\subsubsection{Un exemple quasi unique du genre}

Les semi-distinctifs datent de l'époque charnière entre la disparition des distinctifs et la naissance des cumulatifs. Ils sont assez peu nombreux. En effet, lorsque les synonymistes ont cessé de faire des dictionnaires distinctifs car les cumulatifs représentaient la chance de pouvoir mettre en rapport beaucoup plus de termes, les semi-distinctifs semblaient être en même temps trop distinctifs. Ainsi, les semidistinctifs sont véritablement peu nombreux. Les auteurs de ce type de dictionnaire ont voulu conserver la possibilité d'éclairer le lecteur lorsque la confusion était possible. C'est pourquoi, dans le NDS Larousse (1977), les auteurs mettent le lecteur en garde à l'article « Espion ». Il existe en effet plusieurs sortes d'espions : l'agent secret, l'affidé, le mouchard, l'indicateur, ou encore le mouton. Il ne suffit pas de savoir que tous ces termes sont possiblement substituables à espion, il faut savoir que l'un désigne la personne qui dénonce, l'autre celle qui renseigne les policiers, l'autre est un homme de confiance, etc. Le dictionnaire semi-distinctif renseigne donc le lecteur sur les contextes d'énonciation.

\subsection{Les cumulatifs}

\subsubsection{Un genre qui s'est imposé}

Les dictionnaires de synonymes cumulatifs sont des dictionnaires qui sont constitués de listes. À la suite de l'entrée, il y a une liste de termes qui sont synonymes du mot-vedette. Cependant, les degrés de synonymie qu'il y a entre ces termes ne sont pas indiqués, ni, surtout, les différences qui les séparent. Les explications sont quasi nulles. Seules parfois peuvent apparaître des précisions telles que des marques de registres de langue. Mais pour les dictionnaires de synonymes cumulatifs, les précisions ne vont pas plus loin. Il n'y pas d'exemples pour illustrer dans quelles conditions le terme s'emploie. L'avantage de ce type de dictionnaire est qu'il laisse le choix au lecteur de sélectionner lui-même le terme qui convient le mieux selon le contexte. Cependant, le lecteur n'est pas informé des différentes acceptions du mot, et donc ce n'est pas suffisamment clair pour faire le bon choix. Ouvrir un dictionnaire de synonymes, c'est comme ouvrir un dictionnaire bilingue : tous les termes proposés sont potentiellement justes, mais sans le contexte il est difficile de choisir le bon mot. Ce qui manque donc à ce genre de dictionnaires, c'est le contexte .

\subsubsection{Des dictionnaires très fortement représentés}

Il est très difficile de répertorier les dictionnaires cumulatifs. En effet, aujourd'hui tous les dictionnaires de synonymes présents sur le marché sont des cumulatifs, que se soit des dictionnaires papiers ou éléctroniques. Ainsi, toutes les grandes maisons d'édition comme Larousse, Le Robert, Hachette, etc. comptent dans leur catalogue des dictionnaires de synonymes, tous cumulatifs.

Le centre de recheche CRISCO a constitué un dictionnaire de synonymes de 49000 entrées et 396000 synonymes, soit en moyenne 8 synonymes par termes. Or avec 396000 synonymes, il semble impossible d'avoir autant de définitions que de synonymes. Bien que ce dictionnaire soit électronique et qu'il bénéficie à ce titre de contraintes éditoriales moins rigides ${ }^{10}$, il ne comprend pas de définitions. Cependant, ce dictionnaire étant hébergé sur le portail électronique du CNRTL, il bénéficie du TLFi. Ainsi, le lecteur peut jouir de la définition du TLFi et ainsi avoir toutes les informations nécéssaires pour faire le bon choix. 
Pour la constitution de notre corpus, nous avons choisi de façon tout à faire arbitraire le dictionnaire de Boussinot. En effet, tous les dictionnaires cumulatifs étant fait sur le même modèle, nous aurions pu choisir n'importe quel dictionnaire de cette catégorie pour notre démonstration ${ }^{11}$.

Le format des dictionnaires papiers peut expliquer le manque d'informations distinguant les termes. Ils sont en effet assez petits (environ 500 pages), loin des tailles des dictionnaires généraux. Nous pourrions penser que ce format est fait pour que le dictionnaire soit plus facilement transportable. Cependant, les dictionnaires de synonymes en ligne ont la même approche, c'est-à-dire que nous ne trouvons pas plus de définitions que sur papier. Des dictionnaires en ligne tels que www.synonymie.com, ou www.dictionnairesynonymes.com, ne proposent que des listes de synonymes, comme les dictionnaires cumulatifs papiers. Or, sur la toile, il serait tout à fait envisageable de donner des définitions car les contraintes éditoriales sont plus permissives. En effet, les auteurs n'ont pas un nombre de pages à respecter, donc il serait plus agréable, pour faire le bon choix, que les dictionnaires de synonymes en ligne optent pour la méthodologie de la distinction. Ce n'est pourtant pas le cas. Cela signifie donc que c'est une volonté de la part des synonymistes d'aujourd'hui de ne pas apporter de précisions aux synonymes qu'ils distinguent. En effet, de nos jours, tout le monde possède un dictionnaire général. Ainsi, le lecteur peut consulter les définitions dans les dictionnaires de langue ou encyclopédique afin de déterminer le mot le plus adapté à sa recherche.

\subsection{Quelques informations}

Afin d'avoir une vision matérielle des dictionnaires en question, nous avons consigné ci-dessous dans un tableau le nombre de pages et d'entrées de chaque dictionnaire du corpus. Précisons que pour les dictionnaires de Sardou, le NDS Larousse (1977) ainsi que le dictionnaire de Boussinot, nous n'avons comptabilisé que les entrées qui n'étaient pas uniquement suivies de renvois.

\begin{tabular}{|c|c|c|c|c|c|c|}
\hline & Girard & Roubaud & Guizot & Sardou & NDS Larousse & Boussinot \\
\hline Pages & 359 & 2026 & 986 & 580 & 446 & 890 \\
\hline Entrées & 296 & 573 & 1341 & 1432 & 3562 & 7476 \\
\hline
\end{tabular}

Tableau 1 : Nombre d'entrées et de pages des différents dictionnaires.

Nous pouvons ainsi constater que nous sommes en présence de dictionnaires dont le nombre de pages ou d'articles est très varié. Roubaud dans le double de pages compose pas loin de quinze fois moins d'articles que Boussinot ce qui tient évidemment au type d'article. En effet, ne donnant aucune définition ni aucun exemple, Boussinot peut évoquer un plus grand nombre de termes synonymes que Roubaud.

Nous avons ensuite voulu observer le degré moyen de synonymes par entrées. Les différences sont ainsi énormes.

\begin{tabular}{|l|c|c|c|c|c|c|}
\hline & Girard & Roubaud & Guizot & Sardou & NDS Larousse & Boussinot \\
\hline Degré moyen & 2,41 & 2,58 & 2,58 & 2,59 & 7,46 & 12,98 \\
\hline
\end{tabular}

Tableau 2 : Degré moyen de synonymes

Ainsi, nous constatons véritablement la différence entre les différents types de dictionnaires évoqués ici. En effet, les distinctifs ne comportent pas plus de 2,59 termes synonymes par distinction, tandis que les cumulatifs peuvent en avoir pas loin de 13. C'est véritablement du nombre de mots traités par entrées que la différence est très importante entre ces dictionnaires. Au XVIII ${ }^{\mathrm{e}}$ siècle, on n'a pas le choix entre beaucoup de synonymes lorsqu'on consulte un dictionnaire de synonymes, tandis que de nos jours, le choix est tel que sans définition, il est difficile d'opter pour le bon mot.

Nous avons enfin voulu récapituler les différents traits des dictionnaires de synonymes de la façon suivante : 


\begin{tabular}{|c|c|c|c|c|}
\hline & Distinctifs & Cumulatifs & Semi-distinctifs & Compilation \\
\hline Définitions & + & - & $+/-$ & + \\
\hline Exemples & + & - & + & + \\
\hline Citations & + & - & - & + \\
\hline Listes & - & + & $+/-$ & - \\
\hline Explications & + & - & $+/-$ & + \\
\hline Entrées multiples & + & - & - & + \\
\hline Un seul mot-vedette & - & + & + & - \\
\hline
\end{tabular}

Tableau 3 : Les différents traits des dictionnaires de synonymes

À la vue de ce tableau, nous pouvons tirer quelques conclusions :

- Les traits retrouvés dans les dictionnaires distinctifs et les compilations sont identiques. En effet, il est alors visible que la compilation est un genre particulier des distinctifs.

- Les traits retrouvés dans les semi-distinctifs sont véritablement la somme des traits que l'on trouve dans les distinctifs et les cumulatifs.

- Enfin, les cumulatifs et les distinctifs, bien loin d'être de simples genres différents, sont des genres opposés. En effet, il n'y a aucun trait en commun entre les deux genres.

Nous en arrivons donc à la conclusion suivante: il existe et a existé trois genres différents de dictionnaires de synonymes comme nous le constatons grâce à l'étude de la macrostructure des différents dictionnaires étudiés. Le NDS Larousse (1977) est donc véritablement un sous genre. Nous allons pouvoir désormais observer les différentes informations qui composent ces dictionnaires afin de voir s'il existe d'autres sous catégories.

\section{4 Évolution d'un genre, des dictionnaires qui n'apportent plus les mêmes informations}

\subsection{Les marques d'usage}

Les marques d'usage sont très peu présentes dans les dictionnaires distinctifs. À aucun moment Girard ne fait de distinction entre des termes de registres de langue différents. En effet, il ne figure pas de mention « familier », ou « soutenu » puisque l'auteur n'emploie que des termes de registre courant, voire légèrement soutenu. Ceci semble logique pour l'époque, car, au XVIII ${ }^{\mathrm{e}}$ siècle, on ne peut pas prôner l'emploi d'un terme familier. Ainsi, les mots qu'il met en vedette pour les comparer ne sont que d'un certain niveau de langue propice en ce siècle. Le but de Girard est donc de ne proposer que des termes que l'on pourra réutiliser en société étant donné que l'intérêt de ce dictionnaire est d'insister sur les nuances qui se trouvent entre les termes afin de savoir lequel utiliser. Il est donc très intéressant de constater qu'en 1718 le synonymiste ne considère pas utile, ni même convenable, de se pencher sur des termes familiers, peu soutenus. D'ailleurs, dans le discours préliminaire de son ouvrage, il explique qu' « il est bien difficile qu'un langage fade et grossier vienne d'un esprit fin $»^{12}$.

Au début du siècle suivant Guizot, n'emploie pas non plus de terminologie telle que «familier » ou " soutenu » car il s'attache dans ses propres articles à définir le rôle des personnes portant le nom. Ainsi, pour l'article « Adroit, Industrieux, Ingénieux », il commence ainsi : 
Un homme ingénieux imagine, un homme industrieux trouve les moyens d'exécuter ; un homme adroit exécute. Le dernier met en pratique les inventions du premier et les théories du second.

Guizot (1809 : 23-24)

Il cherche à comprendre ce qui distingue en pratique les termes, et ne recherche pas à les comparer dans leurs emplois mais dans la fonction qui leur est propre.

Sardou s'intéresse plus que ses collègues aux différents sens d'un terme, aux circonstances qui poussent à l'emploi d'un mot plutôt qu'un autre. Lorsqu'il compare des termes, il reste dans la même lignée que ses collègues en ne comparant que des mots " corrects " pour l'époque. Ainsi, il met dans la même entrée « Animal, Bête et Brute (au figuré), Stupide, Imbécile, Idiot», mais n'ajoute pas des termes plus familiers comme « con », « nouille », ou « gland » et « tête de nœud » comme on les trouve dans le dictionnaire de Bertaud du Chazaud à l'article "Imbécile». Bien sûr, Sardou ne peut pas employer ces termes qui n'existaient pas à l'époque, mais nous constatons qu'il ne met pas de termes familiers quels qu'ils soient.

$\mathrm{Au}$ début des dictionnaires de synonymes, lorsqu'ils étaient pourtant distinctifs, le but n'était pas de comparer des termes de registres différents, ni de mentionner tous les termes substituables à un autre qu'ils soient d'un registre soutenu ou vulgaire. À cette époque, n'étaient mentionnés que les termes d'un certain niveau de langue acceptable. En revanche, dans ces dictionnaires il y a des références au type de langage utilisé :

Roubaud (1796) : «Langage de la jurisprudence $»^{13}$, «Langage des précieux $»^{14}$, « Langage baroque $»^{15}$, «Langage des physiciens »" ${ }^{16}$, Dans le langage de l'économie rurale ».

Guizot (1809) : «Un boucher peut dire $»^{17}$, « Dans le langage didactique de la physique $»^{18}$, « Dans le langage de la religion $»^{19}$.

Ou encore Sardou (1866) : « Dans le langage féodal $»^{20}$.

Il y a aux XVIII ${ }^{\mathrm{e}}$ et XIX ${ }^{\mathrm{e}}$ siècles un désintérêt total pour les registres de langue dans les dictionnaires de synonymes sauf lorsqu'il s'agit d'un type de langage propre à une profession ou une religion. Il faut donc comparer cela aux dictionnaires des $\mathrm{XX}^{\mathrm{e}}$ et $\mathrm{XXI}^{\mathrm{e}}$ siècles afin d'observer si désormais les registres de langue peuvent être considérés comme un critère de différenciation entre les termes synonymes, s'il y a une différence entre les dictionnaires distinctifs et les dictionnaires cumulatifs.

Il suffit de lire la préface du NDS Larousse (1977) pour savoir qu'à cette époque, la distinction selon les registres de langue n'était pas habituelle car il y est précisé :

Nous avons [...] signalé telle locution familière généralement absente des dictionnaires de ce genre.

NDS Larousse (1977 : 5)

Ainsi, les registres de langue n'étaient pas jugés comme substituables jusqu'au milieu du $\mathrm{XX}^{\mathrm{e}}$ siècle lorsqu'il s'agissait de synonymie. En effet, les registres familiers n'étaient pas considérés comme employables, et donc pas mentionnés en tant qu'alternative à un autre terme. Persiste le sentiment qu'un terme familier ne doit pas être employé, il faut lui préférer un terme soutenu, ou courant. Désormais les synonymistes consignent dans leurs dictionnaires des termes autres que courants, ou du bon usage. À l'intérieur de l'article, l'auteur indique quand le terme proposé est d'un certain registre. Ainsi à l'article « Cuisine » se trouvent les mentions : « popote» (familier) ou « bouffe » (très familier). Par contre, il n’y a pas d'entrée «Bouffe». L'auteur se contente d'indiquer en tant que synonyme ce terme familier, mais ne se risque pas encore à le mettre en tant que vedette. Trente ans plus tard, Bertaud du Chazaud utilisera la vedette « Bouffe ». Le NDS Larousse (1977) n'est donc qu'au commencement de l'acceptation des termes non courants dans un dictionnaire de synonymes.

Prenons l'exemple d' « Occasion ». Parmi la longue liste de synonymes que propose Bertaud du Chazaud dans son dictionnaire, il y a « chopin » (argot), « occase » (familier) et « rossignol » (péjoratif). Il n'est pas envisageable de trouver ces termes dans le dictionnaire de Girard qui, lui, mentionne comme 
synonymes de ce mot, « circonstance » et « conjonction ». Ces renseignements qu'apporte Bertaud du Chazaud précisent qu'il n'est pas possible de dire « occase », à quelqu'un qui ne nous est pas familier.

Enfin, en ce qui concerne la manière de consigner les termes de différents registres, le Dictionnaire de Synonymes et Nuances et le dictionnaire de Boussinot ne diffèrent pas de celui de Bertaud du Chazaud. En effet, les trois ouvrages mentionnent les termes « péjoratifs », « populaire » ou « vulgaire » aussi bien dans les entrées que dans le corps de l'article. Ce qui signifie bien que le NDS Larousse (1977) a ouvert une voie en 1977 qui sera reprise par tous les dictionnaires de synonymes à venir, mais aussi, que non seulement les termes non courants sont considérés comme acceptables et comme substituables à un terme $\mathrm{du}$ registre courant, mais également que ce sont des termes que l'on peut chercher directement dans le dictionnaire. Ceci témoigne d'une importante évolution.

\subsection{L'étymologie}

L'étymologie est un sujet de débat dans le monde de la synonymie au XVIII ${ }^{\mathrm{e}}$ siècle. En effet, en ce qui concerne l'étymologie, les avis sont très partagés. Roubaud, qui est le premier auteur à utiliser l'étymologie dans son dictionnaire, en use à outrance. En effet, pour tous les termes il donne l'étymon, or celui-ci est de façon très régulière celte. Cela provient d'un courant de pensée de l'époque que l'on doit à Court de Gebelin qui veut que la langue française provienne de la langue celte comme le souligne Guizot dans son introduction « [Court de Gebelin] fait du Celtique la source de toutes les langues européennes, anciennes ou modernes, et mêmes de plusieurs langues de l'Asie occidentale » (Guizot, 1809 : xxxij)

Girard, ne mentionne pas l'étymologie dans son ouvrage, il ne cherche pas l'origine des termes, il préfère se baser uniquement sur la définition.

Guizot, Morin, Sardou et Laveaux s'accordent à dire que le recours à l'étymologie est très intéressant, instructif. Cependant, ils ne s'en servent pas, et même, les compilateurs retirent des extraits empruntés à Roubaud toute allusion étymologique. Seul Morin conserve un peu d'étymologie de Roubaud.

Nous pouvons donc dire, que bien que Guizot affirme dans sa préface que l'étymologie est importante dans l'étude des synonymes, tout comme Sardou qui conseille d'examiner l'étymologie, les compilateurs font le choix de supprimer cette partie lors de leur retranscription du dictionnaire de Roubaud. Pourquoi ? En effet, il y a visiblement à l'époque un intérêt certain pour l'étymologie, mais seul Roubaud semble se lancer dans l'étude de celle-ci.

En effet, au XVIII ${ }^{\mathrm{e}}$ siècle, l'analyse étymologique du français fait un écart par rapport à la tradition qui veut que la langue française vienne du latin. Écart peu convainquant, mais avec quelques adeptes. Au moment où l'on commence à voir que les mots français ne viennent pas spécialement du latin des érudits, mais plutôt du bas latin, un certain nombre de gens ont préféré croire que le français venait en partie du celte. Ce qui sera le cas de Roubaud, disciple de Court de Gebelin. C'est pourquoi les compilateurs contemporains de Roubaud ne l'ont pas suivi dans ses explications car il faisait fausse route.

$\mathrm{Au} \mathrm{XX}^{\mathrm{e}}$ siècle, l'attitude des lexicographes synonymistes est tout à fait différente concernant l'étymologie. En effet, plus aucun dictionnaire de synonymes, qu'il soit cumulatif ou semi-distinctif, n'aborde le sujet de l'étymologie. Ce qui est dû à la structure du dictionnaire. Le dictionnaire cumulatif étant constitué de listes ne laisse pas la place à des données étymologiques. Le dictionnaire de synonymes s'est spécifié au fil des siècles. Désormais lorsqu'on consulte un dictionnaire de ce type, on trouve une liste de termes accompagnant le mot-vedette. Si l'on cherche l'étymologie d'un terme de la liste, il faut consulter un dictionnaire général ou étymologique. Ainsi, les dictionnaires de synonymes actuels, sont de véritables dictionnaires de synonymes ne traitant que de synonymie et non de sujets annexes. En effet, même si c'est grâce à l'étymologie que le synonymiste peut comparer certains termes, le lecteur quant à lui, ne cherche pas à savoir la raison pour laquelle les termes sont voisins. Ainsi, l'étymologie n'a plus sa place dans les dictionnaires de synonymes actuels. 


\subsection{Les définitions}

Précisons tout d'abord que les définitions des dictionnaires de synonymes distinctifs ainsi que des semidistinctif, sont accompagnées (à l'exception du dictionnaire de Girard) d'informations catégorielles. Cependant, depuis les cumulatifs, il n'y a plus de renseignements de ce genre.

En ce qui concerne les définitions, il est très facilement visible que les synonymistes n'ont pas la même attitude au XVIII ${ }^{\mathrm{e}}$ et $\mathrm{XXI}^{\mathrm{e}}$ siècle. En effet, avant même de lire le dictionnaire, uniquement en le feuilletant, nous constatons que les définitions ont changé puisqu'au XVIII il y en a, et aujourd'hui il n'y en a pas.

$\mathrm{Si}$ aux $\mathrm{XX}^{\mathrm{e}}$ et $\mathrm{XXI}^{\mathrm{e}}$ siècles, tous les dictionnaires de synonymes sont construits sur le même modèle (c'est-à-dire des listes ou des listes accompagnées d'exemples pour les semi-cumulatifs), au XVIII ${ }^{\mathrm{e}}$ siècle, il y eut deux écoles. D'un côté celle de Girard, de l'autre celle de Roubaud. Cependant, celles-ci repésentent deux extrêmes et ne sont suivies par personne.Tous les synonymistes se situent entre les deux fondateurs.

Girard compose de façon très simple son dictionnaire. Il se contente de donner une définition pour les mots-vedettes. Il ne va pas plus loin. Chaque terme est défini, et ainsi comparé à un autre.

Roubaud, étoffe son dictionnaire car il donne l'étymologie (comme signalé précédemment), mais aussi des définitions, des descriptions, des citations, des exemples. Soulignons, pour se rendre compte de l'ampleur des définitions du dictionnaire de Roubaud, que Guizot consigne le double de termes dans moitié moins de pages que Roubaud.

La quasi-totalité des synonymistes venant après Roubaud se trouve à mi-chemin entre la manière de procéder de Girard et celle de Roubaud. En effet, loin de se contenter de donner une définition pour chaque terme comme le fait Girard, ils n'approfondissent pas autant les descriptions que Roubaud. Cependant, ils adoptent sa méthodologie d'apporter comme arguments à la comparaison des citations et exemples, tout en retirant les descriptions subjectives et les étymologies foisonnantes.

Ainsi, la différence définitionnelle des synonymistes implique une différence dans la consultation du dictionnaire. En effet, de nos jours on consulte un dictionnaire de synonymes lorsqu'on ne retrouve pas le terme cherché. Le dictionnaire de synonymes, grâce aux listes de termes qu'il comporte est alors suffisant. Cependant, lorsqu'on n'a pas la connaissance nécessaire, le dictionnaire général est obligatoire afin de savoir quel mot sera le plus approprié.

À notre époque, si l'on cherche les ressemblances ou différences existantes entre des termes considérés comme synonymes d'après le dictionnaire de synonymes consulté, il faut avoir un dictionnaire général à portée de main. Ainsi, si l'on cherche « orgueil » dans le dictionnaire de Boussinot, il y a une liste de 17 termes pouvant être synonymes. Il faudra donc, pour une personne ne connaissant pas parfaitement les nuances de chacun de ces termes consulter un dictionnaire général pour savoir ce qui diffère entre l'arrogance, l'insolence, la prétention, l'importance, la suffisance, la fierté ou encore la vanité. Tandis que dans le dictionnaire de Girard, il est indiqué que « l'Orgueil fait que nous nous estimons ; la Vanité fait que nous voulons être estimés. ${ }^{21}$ L'absence de définitions dans les dictionnaires de synonymes contemporains oblige le lecteur à avoir un dictionnaire général à ses côtés lors de ses recherches.

\subsection{Récapitulatif des différentes informations}

De la même façon que nous avions rassemblé les traits caractéristiques des différents dictionnaires de synonymes, nous avons établi un tableau récapitulatif des types d'information que nous trouvons dans ces dictionnaires.

\begin{tabular}{|c|c|c|c|c|c|c|}
\hline & Girard & Roubaud & Guizot & Sardou & NDS & Boussinot \\
\hline Étymologie & - & + & + & $+/-$ & - & - \\
\hline
\end{tabular}




\begin{tabular}{|c|c|c|c|c|c|c|}
\hline Définitions & + & + & + & + & $+/-$ & - \\
\hline Catégorisation & - & + & + & + & $+/-$ & - \\
\hline Exemples & + & + & + & + & + & - \\
\hline Citations & - & + & + & + & - & - \\
\hline Registres & - & - & - & - & + & + \\
\hline Différenciation de sens & - & - & - & - & + & + \\
\hline
\end{tabular}

Tableau 4 : Les différences types d'informations

Ainsi nous constatons plusieurs choses :

- Le dictionnaire de Boussinot ne comporte aucune information présente dans les dictionnaires distinctifs. Ce qui confirme alors que les distinctifs et les cumulatifs ne sont pas opposés que dans la forme qu'ils ont, mais aussi dans les informations qu'ils apportent.

- Seul à partir de l'époque des cumulatifs et des semi-distinctifs, on a commencé à trouver des distinctions faites à partir des différents contextes et sens du terme.

- Et enfin, comme nous l'avions déjà constaté, le semi-distinctif se trouve encore entre les deux genres puisqu'il donne aussi bien des informations présentent dans les distinctifs que dans les cumulatifs, mais aussi car il n'y a pas toutes les informations rencontrées dans les distinctifs.

Ce tableau conclu parfaitement notre article puisque nous constatons qu'il y a une forte évolution du genre des dictionnaires de synonymes à travers les siècles, et que désormais, les synonymistes ne donnent que des renseignements nécessaires et indispensables à ce genre lexicographique tandis qu'auparavant, ils se laissaient plus guidés par les raisons de différenciation et d'illustration.

\section{Conclusion}

Le dictionnaire de synonymes est un genre de dictionnaire qui a connu une grande évolution à travers les siècles. Il voit le jour suite à un constat : certains termes peuvent paraître identiques. Cependant, il existe une différence aussi minime soit elle qui oblige le locuteur à être attentif car il ne faut pas employer un terme pour un autre. Les premiers dictionnaires permettent donc d'offrir à leur public un ouvrage certes peu fourni dans le nombre de mots-vedettes, mais très complet quant aux définitions, rapprochements étymologiques ou encore explications morphologiques. De nos jours, les synonymistes procèdent de façon inverse, ils couvrent leurs ouvrages de mots-vedettes et de listes qui restent inexpliquées, mais les dictionnaires n'apportent aucune information quelle qu'elle soit.

Ainsi, puisque les synonymistes d'aujourd'hui n'offrent plus les mêmes dictionnaires que leurs collègues du siècle dernier, les utilisations ont changé. En effet, n'ayant point d'explications sur le sens exact d'un terme, l'usager d'un dictionnaire de synonymes, de nos jours, ne peut se passer d'un dictionnaire général lors de sa consultation des synonymes, sauf s'il ne désire connaître que les degrés de registre de langue d'un terme. De nos jours les dictionnaires de synonymes sont en effet d'excellents outils pour connaitre les marques d'usage d'un mot. Nous pouvons donc nous interroger pour savoir s'il ne serait pas possible, pour les dictionnaires éléctroniques par exemple, d'éttofer les définitions afin que le lecteur ne soit pas dans l'incertitude lorsqu'il cherche un synonyme.

\section{Bibliographie}

Bertaud du Chazaud, H. (2003). Dictionnaire de synonymes et mots de sens voisins. Paris : Gallimard. 
Boussinot, R. (2007). Synonymes, analogies, antonymes. Réédition sous la direction de Pruvost, J. Paris : Bordas.

García Platero, J.-M. (2008). La sinonimia en el diccionario. Problemas de codificación. El Diccionario como puente entre las lenguas y culturas del mundo, Alicante: Universidad de Alicante. pp. 352-356.

Genouvrier, É. (dir) (1977). Nouveau Dictionnaire des synonymes. Paris : Larousse.

Girard, G. (1718). La Justesse de la langue françoise. Édition consultée : édition annotée par Maria Gabriella Adamo, 1999, Fasano : Schena Editore.

Guizot, F. (1809). Nouveau Dictionnaire universel des synonymes. Paris : Aimé Payen.

Laveaux, J.-C. (1826). Dictionnaire synonymique de la langue française. Paris : Ledentu fils.

Le Fur, D. (dir.). (2005). Dictionnaire des Synonymes et nuances. Paris : Le Robert.

Lévizac, J.-P. V. De. (1807). Dictionnaire universel des synonymes de la langue française. Londres : Cox fils et Baillys.

Montmeran, A. de. (1645). Synonimes et épithètes françoises recueillies et disposées selon l'ordre de l'alphabet. Paris : Le Bouc.

Morin, B. (1801). Dictionnaire universel des synonymes. Paris : Lesguilliez frères.

Pruvost, J. (2006). Les Dictionnaires français, Outils d'une langue et d'une culture. Paris : Ophrys.

Roubaud, P. (1785). Nouveaux synonimes français. Paris : Moutard.

Roubaud, P. (1796). Nouveaux synonimes français. Paris : Moutard.

Sardou, A.-L. (1857). Nouveau Dictionnaire des synonymes français. Édition consultée : réédition de 1866. Paris : Delagrave.

Stevens, A. (2000). L'Ontologie d'Aristote au carrefour du logique et du réel. Vrin : Fondation universitaire de Belgique.

Vivre, G. de. (1569). Synonimes, c'est à dire plusieurs propos propres tant en escrivant qu'en parlant, tirez quasi tous à un mesme sens, pour monstrer la richesse de la langue françoise. Cologne : Von Aich.

Internet :

Dictionnaire des synonymes et des antonymes français. $<$ http://www.synonymie.com> (consulté le 2 janvier 2010)

Dictionnaire des synonymes. $<$ http://www.dictionnaire-synonymes.com> (consulté le 2 janvier 2010)

Centre national de ressources textuelles. $<$ http://www.cnrtl.fr $>$ (consulté le 2 janvier 2010)

$1 \mathrm{Cf}$. Annexe 1.

${ }^{2}$ Justesse de la langue françoise, 1781.

${ }^{3}$ Nouveaux synonimes françois, 1785.

${ }^{4}$ Cf. Annexe 2.

${ }^{5}$ Avertissement, page 1.

${ }^{6} \mathrm{Cf}$. tableau 1 .

${ }^{7}$ Appelé désormais NDS Larousse.

${ }^{8}$ Cf. Annexe 3.

${ }^{9}$ Cf. Annexe 4.

${ }^{10}$ Dans un dictionnaire papier, l'auteur est confronté à un problème : le nombre de pages à respecter. En effet, le dictionnaire ne peut dépasser un nombre de pages raisonnable (pour que le dictionnaire soit transportable et peu coûteux).

${ }^{11}$ C'est pourquoi dans cet article nous évoquerons d'autres dictionnaires cumulatifs qui ne sont pas dans le corpus. 


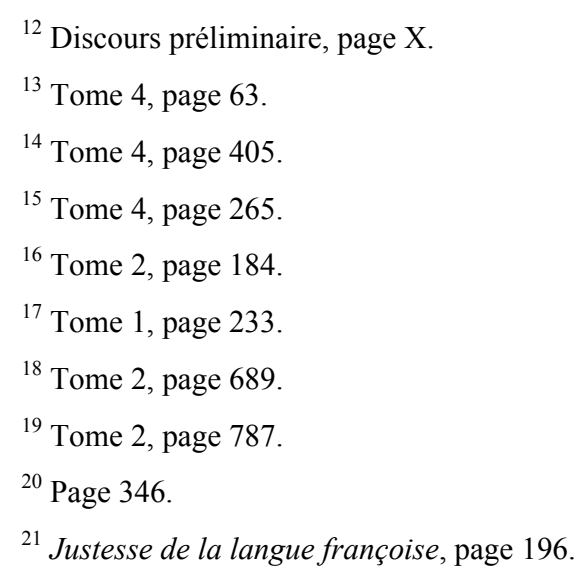

\section{Annexes}

138 STNONYMES FRANÇOIS].

$$
\text { Afyle, Refuge. }
$$

Lieux où l'on fe met en fûreté, à l'abri, couvert.

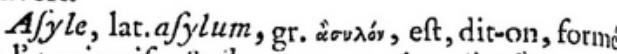
de l'a privatif, \& de suג»s, je tire, j'enleve; car il n'étoit pas permis d'arracher quelqu'un d'un afyle. Ce mot vient de l'oriental afcher, atfer, afel, bois, bois facré ; d'où $\int y l$-va, bois. Tfel fel, fignifie ombrage : le bois prête de l'ombrage. Il n'y avoit point d'a fyle facré fans bois, ou plutiôt le bois étoit l'afyle proprement dit. L'afyle établi par Romulus étoit un bois de chêne, \& s'appeloit quercetum. Refuge vient du latin fugere, fuir refuggere, fuir à pas redoublés, avec perfévérance, par crainte, par horreur. Le refuge cft l'afyle vers lequel on s'enfuit, dans lequel on fe jette lorf qu'on eft pourfuivi, lorfqu'on veut échapper à un danger preffant.

Ainfi dès qu'on craint un danger, on cherche un afyle: alfailli d'un péril, on cherche un refuge. Il faut un afylo pour le befoin; dans la néceffité, un refuge. On fe retire, on fe jette dans un afyle: on fe jette, on fe fauve dans uni refuse.

Un port eft en tout temps un afyle : dans la tempere, c'eft un refuge. Le voyageur égaré cher. che un afyle; \& pourfuivi, un refuge. Le refuge fuppofe un grand danger: l'afyle n'en exclut aucun.

Adam, chaffé de l'afyle de l'innocence, ne fçair sì trouver un refuge contre les pourfuires de l'Ánge exterminateur : Quò à facie tuâ fugiam?

Cicéron appeloit lẹ. Sénat Romain l'afyle des

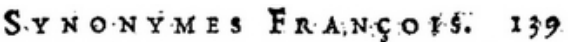
Rois, des Peuples, des Nations : oui, mais ces Nations, ces Peuples, ces Rois avoieunt bientôt befoin de refuge contre la tyrannie de Rome. La Fraice a fouvent été l'afyle des Rois qui ont abandonné le trône, \& le refuge de ceux qui en ont ćté cliaflćs.

On a dir que Cicéron étoit l'a fyle des malheureux, \& Caton le fléau des méchans. Pourfuivez, ii vous le voulez, lés méchans jufque dans leur detnier refuge avec Caton; je refterai auprès de Cicéron.

L'excommunication, chez divers Peuples païens, eil fermant tout afyle aux profcrits, ne leur laiffoit pour refuge que le défert ou l'humanité d'un cour clevé au.deffus de la fuperfition.

L'afyle facré ne peut être que pour l'innocence; car vous ne ferez pas fervir la Divinité à protéger $\delta$ 'à encourager le crime par une impunité funeite. Aufi n'étoit-il point, chez les Juifs, un refuge pour un criminel pourfuivi par le júgement même de la Loi.

Le favori d'Arcadius., le premier qui fit abolir le droir d'afyle, ne tarda point à chercher un refuge contre la mauvaife fortune.

Aux malheureux, des afyles; pour les malfaiteurs, point de refuge. Que dis-je ? la Juftice, elle-même, cft leur refuge, puifqu'en mêtme temps qu'elle les fouftrait aux viengeances particulieres \& atx peines démefurćes, elle n'a pour but que de réparer leurs torts, \& de les ramener à leur devoir; E la miféricorde ef toujours avec elle. -Préparons nous un afyle dans notre propre caur, î un refuge daiss les bras de la Providence. 
140 ŜXNONYMES FRANÇOTs: La mort eft l'afyle de la vieilleffe, \& le refuge du défépoir.

Le jufte a befoin d'afyle, car il a toujours i craindre; le pécheur a befoin de refuge, car il eft toujours menacé \& pourfuivi, du moins pat $f_{a}$ confcience.

M. l'Abbé Poule dir du vrai Chrétien, dans fon Sermon fur la Foi, qu'il eft l'afyle de la veuve \&ै de l'orphelin, \& un refuge de miféricorde. No: feulement il défend le foible contre le danger, mais il l'en préferve; \& par-lì il eft fon afyle. Comme Dieu lui-même, il ne veut point la mott du pécheur, il veut $\mathfrak{f}_{a}$ converfion; \& par-là il eft un r'fuge de miféricorde.

Il n'y a d'afyle sûr, dit Rouffeau, que celui où l'on peut échapper à la honte \& au repentir. Il n' a point de refiuge pour qui voudroit échapper à foi-même, fi ce n'eft la Religion.

Le travail eft l'a/yle naturel du pauvre : la charité fera fon refuge dans fes infirmités: Mais fongeons aux fervices nombretx que l'homme in. valide peut encore rendre à $\mathrm{fa}$ famille, s'il ne lui eft point à charge ; \& qu'il eft mort pour elle, fi on l'en fépare. L'empire de la Nature \& de la raifon n'a pas befoin d'une force étrangere.

Lorfque le pieux Cordelier Jean Tifferand ot: vrit un refuge aux femmes de mauvaife vie, plus de fix cents Pénitentes s'y jetterent tout d'un coup, qui furent bientôt fuivies d'une foule prodigieufe. Il ne leur avoit donc manqué qu'un afy-le pous préferver leur innocence?

Les Loix font notre afyle; nots vivons en füretć fous leur fanve-garde. Le fanokuaire de la Juftice
SYNONYMEG FRANÇOI'S: I4I eft notre refuge; mais sil eft ou inacceffible ou impénétrable, quelle reflource!

Les Romains envient à l'indomptable Annibal lafyle qu'ils lui ont laiffé dans fa parrie. Pourfuivi por eux, il croit trouver un refuge chez les Rois, conme fi le caur des Rois étoit le refuge de la droiture.

Le mot d'a fyle ćtant particuliérement deftiné idéligner l'a fy le facré, il rappelle l'idée fpéciale de fareré, de fauve-garde, de force, d'efficacité : cette idćen'eft pas fi eflentielle au refiuge. L'afyle eft fait on difpofé pour nons mettre à labri ; c'eft notre rempart : le refuge nous mettra peut-être à couvert, mais plutôt par accident; c'eft notre retraite. Dans l'afyle, on eft hors de danger : dans le refuge, on tíchappe fouvent qu'à la pourfuite. Vous ne ferez pus arraché de votre $a / y$ le : on pourra vous arracher de votre refuge, fi on le découvre. Les puiffans vousdonnent afyle: le pauvre vous offrira un pauvre refuge. Le premier lieu qui vous dérobe à vos perfecuteurs, eft votre refuge; $s^{\prime}$ il ne vous défend pas contre eux, ce n'elt point un afyle.

Par la nêtne raifon, l'a fyle ne fe prend que pour une retraite honncte \& refpectable; \& il nen et pas de même du refuge. $\mathrm{L}_{a}$ folitude eft un afyle pour les contemplatifs : les brigands ont des refuges, comme les bêtes féroces. Les réduits où saffemblent des jouteurs, des vagabonds, des fainicans, s'appellent des refiuges \& non des afyles.

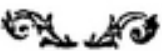

Annexe 1 : Exemple d'article du dictionnaire de Roubaud 


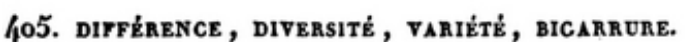
La difference suppose une comparaison que l'esprit fait des choses, pour en avoir des idées précises qui empéchent la confusion. La diversite suppose un changement que le goût cherche dans les choses, pour trouver une nouveauté qui le flatte et le réveille. La varíté suppose une pluralité de choses non ressemblantes que limagination saisit, pour se faire des images riantes, qui dissipent lennui d'une trop grande unifor-
mitê. La bigarrure suppose un assemblage mal assorti, que le mité. La bigarnure suppose un assemblage mal assorti, que le caprice forme pour se réjonir, ou que le mauvais goút adopte. Ia différence des mots doit servir à marquer celle des idées.
Un peu de diversite' dans les mets ne nuit pas à l'économie de la nutrition du corps humain. La nature a mis une variété infinie dans les plus petits objets; si nous ne l'apercevons pas, c'est la faute de nos yeux. La bigarrare des couleurs et des ornemens, fait des habits ridicules ou de thétre. (G.)

406. DIFFÉRENCE, INÉGALITÉ, DISPARITÉ.

Termes relatifs à ce qui nous fait distinguer de la supériorité ou de l'infériorité entre des étres que nous comparons.

Le terme diff'rence s'étend à tout ce qui les distingue; c'est un genre dont linégalité et la disparité sont des especes. L'inégalite semble marquer la différence en quantité; et la disparité, Ga difference en qualité. (Encycl. IV, io37.)

407. DIFFÉRENT, DISPCTE, QUERELLE.

La concurrence des intérêts cause les différens. La contrariłté des opinions produit les disputes. Liagreur des esprits est la source des querelles.

On víde le different. On termine la dispute. On appaise la querelle.

L'envie et l'avidité font qu'on a quelquefois de gros diffetrens pour des bagatelles. L'entétement, joint au défaut d'attention a la juste valeur des termes, est ce qui prolonge ordition a la juste valeur des termes, est ce qui prolonge ordi-
nairement les disputes. Il y a dans la plupart des querelles plus d'humeur que de haine. (G.)

\section{DIFFÉRENT, DÉMÊLÉ.}

Le sujet du diffirent est une chose précise et déterminée sur laquelle on se contrarie, l'un disant oui et l'autre non. Le sujet du démélé est uye chose moins éclaircie, dont on n'est pas d'accord, et sur laquclle on cherche à s'expliquer pour savoir à quoi s'en tenir.
La concurrence cause des differens entre les particuliers. ambition est la source de bien des démélés entre les puissances ( $t$ ). (G.)

409. DIFFICULTÉ, OBSTACLE, EMPËCHEMENT.

La diffic ilse embarrasse; eile se trouve sur-tout dans les affaires, et en suspend la décision. L'obstacle arréte; il se rencontre proprement sur nos pas, et barre nos démarches. Liempechrme at résiste; il semble mis exprès pour s'opposer a exícution de nos volontés.

On dit lever la difficulte, surmonter l'obstacle, ôter ou vaincre l'empechement.

Le unot de difficulted me parait exprimer quelque chose qui nait de la nature et des propres circonstances de ce dont il s'agit. Celui d'obstacle semble dire quelque chose qui vient sagit. Celui d obstacle semble dire quelque chose qui vient
d'unce cause étrangère. Celui l'empechement iait entendre quelque chuse qui dépleud d'une loi, ou d'une force supérieure.

La disposition des esprits fait souvent naitre dans les traités plus de diffic:ltés que la matière méme sur laquelle il est question de statuer. L'éloquence de Démosthènes fut le plus grand obvacle que Philipie de Macédoine trouva dans ses routes politiques, et quil ne put jamais surmonter que par la des armes. La proche parenté est un empechement au mariage que les lois ont mis et que les lois peuvent óter. ( $G$.) 4IO. DIFFORMITÉ, LAIDEUR.

Ces deux mots sont synonymes, en ce quilis sont également opposés à l'idée de la beauté, quand on les applique à la figure humaine.

La difformitéest un défaut remarquable dans les proportions; et la laideur, un défaut dans les couleurs, ou dans la superficio du visage.

(1) En rapprochant cet article du précedent, on n'est pas satisfait mar ce qui distingue le démele et la dispute. Dans lun et dans l'autre, il y' a contrariété d'opinions : la chose n'eat pas d'nccord, et. l'on obershe ì iexpliquer pour savoir à quoi s'en tenir. Quelle est donc la différence de ces deax termes? II me semble qu'clle vient de celle des objets, en ce que la dispute roule sur une matière générale et purement scientilique, et le dismélé sur une matière particulière, et qui peut fonder des prétentions d'intéréts. La dispute s'ćchauffe par le desir de paraítre plus babile; le démelés sanime par le desir de se faire un droit : I'orgueil, qui soutient la dispute, et lavidite, qui est la véritable cause du démêls, font bientot dégénérer l'une en querelle, et lautre en un différent formel. (B.)

Annexe 2 : Exemple de pages du dictionnaire de Guizot 
gosier, kiki. - 3" (dans des express.) Mettre le couteau sous la gorge : v. MENA. CER. A gorge déployée : v. RIRE. Prendre à la gorge : v. SUFFOQUER. Faire des gorges chaudes : v. MOQUER (SE).

II V. BUSTE.

III V. COL.

gorgée Il buvait à grandes gorgées = trait; (fam.) lampée.

gosier V. GoRGE. (fam.) Il a le gosier en pente : il a liquidé toute la bouteille de vin!; (très fam.) dalle.

gourmand $1^{\circ}$ Le gourmand est celui qui aime la bonne chère avec excès et manque de sobriété. Le gourmet est celui qui apprécie la bonne chère et la goûte avec délicatesse et raffinement; (fam.) bec fin et, très fam., fine gueule sont des syn. de gourmet; gastronome implique non seulement le goût, mais l'art de faire bonne chère. $V$, aussi GLOUTON. $-2^{\circ}$ (adj.) V. FRIAND.

goûter I (verbe) $1^{\circ} \sim$ qqch. Goûter un bon vin est pour lui un plaisir incomparable = déguster ; $\uparrow$ savourer ; v. JOUIR et SENTIR I. - $2^{\circ} \sim$ qqch. Je goûte assez peu la musique sérielle (soutenu); (courant) aimer, apprécier; $\uparrow$ raffoler de, être fou de. $-3^{\circ} \sim$ à qqch. Reprenez donc de cette tarte, vous y avez à peine goûté !; (plus fam.) toucher. $-4^{\circ} \sim$ de qqch. Avez-tous déjà goûté de la solitude? (soutenu) = tâter de; (courant) faire l'expérience de.

II (nom) V. COLLATION.

gouvernante Dans les familles riches, l'éducation des enfants est parfois confiée à une gouvernante; la nurse ou bonne d'enfant ne s'occupe généralement que des soins à donner aux enfants en bas âge; la nourrice remplit les mêmes fonctions que la nurse, mais elle n'est pas attachée à une famille (Une famille riche peut se permettre d'avoir une nurse, une famille modeste confie au besoin un bébé à une nourrice, met son enfant en nourrice).

gouvernement $1^{\circ}$ Quel est le type de gouvernement en Australie? = régime (politique). $-2^{\circ} \mathrm{V}$. ETAT IV, POUVOIR III et CABINET I. grâce $11^{\circ} \mathrm{V}$. AMNISTIE. - $2^{\circ} \mathrm{V}$. DON (in donner I). - $3^{\circ}$ (dans des loc. ou express.) Grâce à : v, AIDE (in aider). Faire grâce de : v. PLAISIR. De grâce, laissez-moi tranquille! (très soutenu) $=$ (courant) je vous en prie. Rendre grâce : v, REMERCIER. Crier gráce : v. SUPPLIER. Faire grâce : v, DISPENSER et PARDONNER (in pardon). Gráce à Dieu : v. HEUREUSEMENT. Donner le coup de gráce $=$ achever

II $1^{\circ}$ Il se dégage des gens et des paysages de ce pays une grâce indéniable = eharme (v. ce mot). V. ÉLÉGANCE (in élégant) et LÉGÈRETÉ. - $2^{\circ}$ Il $a$ accepté de bonne gráce votre invitation (soutenu): (courant) volontiers, de bon gré; v. aussi GENTILLESSE (in gentil). Vous auriez mautaise gráce à refuser son intitation! (soutenu) = être mal venu de; (plus courant) être mal placé pour.

grade $1^{\circ}$ V. AVANCEMENT (in avancer II). $-2^{\circ} \mathrm{Il}$ vient d'obtenir le grade de docteur ès sciences; titre évoque davantage le diplôme, grade, la situation sociale à laquelle on accède. $-3^{\circ} \mathrm{En}$ prendre pour son grade : v. RÉPRIMAN. DER.

grand D'emploi très fréquent, cet adjectif admet beaucoup de syn., le plus souvent de sens plus précis ; nous signalons ici les principaux. $1^{\circ}$ (référence à la taille) D'un homme ou d'une femme grands, on dira, fam., que ce sont des asperges, perches, échalas; (un grand) escogriffe ne se dit que de l'homme. $2^{\circ}$ (référence à l'âge) grande personne se dit comme syn. d'adulte quand on parle à des enfants. - $3^{\circ}$ (référence au volume) Faire de grandes dépenses = (postposé) important, considérable; v. aussi EXAGÉRE, FOU et DÉMESURÉ. $4^{\circ}$ (référence à la surface) Une grande plaine; $\uparrow$ vaste; (postposé) étendu. V. AMPLE. $-5^{\circ}$ (adj. à valeur intensive, formant souvent avec le nom qu'il accompagne une express.) Attendre deux grandes heures $=$ long, bon. Employer les grands remèdes $=$ (postposé) extrême. Un grand caur, une grande âme : v. BEAU, NOBLE. Un grand style : v. ÉLE. VE I. Un grand choix : v. VARIÉ (in varier). Elle n'aime que les grandes émotions $=$ (postposé) fort. V. INCOERCIBLE. Un grand chagrin: $\uparrow$ immense ; (postposés) infini, fou. Une grande

Annexe 3 : Exemple de page du NDS Larousse (1977) 


\begin{tabular}{|c|c|}
\hline fort & Malin \\
\hline Vigoureux & Imbattable \\
\hline Robuste & Qualifiant un objet: \\
\hline Puissant & Résistant \\
\hline Herculéen & Dur \\
\hline Ferme & Solide \\
\hline Résistant & Cartonné \\
\hline Solide & Épais \\
\hline Dur & Rigide \\
\hline Athlète & Inusable \\
\hline Athlétique & Renforcé \\
\hline Musculeux & - Antonymes: Faible, Ché- \\
\hline $\begin{array}{l}\text { Musclé } \\
\text { Membru (anc.) }\end{array}$ & tif, Débile, Déficient, Ti- \\
\hline $\begin{array}{l}\text { Membru (anc.) } \\
\text { Vert }\end{array}$ & moré, Peureux, Timide, \\
\hline $\begin{array}{l}\text { Vert } \\
\text { Découplé }\end{array}$ & Craintif, Ignorant, Incom- \\
\hline Trapu & pétent, Nul, Mou. \\
\hline D'acier & forteresse \\
\hline De fer & Fort \\
\hline Invincible & Fortin \\
\hline Incoercible & Citadelle \\
\hline Inflexible & Fortification \\
\hline Costaud (fam.) & Place forte \\
\hline Malabar (pop.) & Abri \\
\hline Bien taillé (ou bâti) & Château \\
\hline Valide & Bastion \\
\hline Moralement : & fortifiant \\
\hline V. COURAGEUX & Réconfortant \\
\hline $\begin{array}{l}\text { Résistant } \\
\text { Armé }\end{array}$ & Reconstituant \\
\hline $\begin{array}{l}\text { Arme } \\
\text { Averti }\end{array}$ & Cordial \\
\hline Énergique & Analeptique \\
\hline Trempé & Remontant (fam.) \\
\hline Solide & Roboratif \\
\hline Ferme & Stimulant \\
\hline Volontaire & Réparateur \\
\hline Autoritaire & Tonique \\
\hline Aguerri & - ANTONYMES: Anémiant, \\
\hline $\begin{array}{l}\text { Intellectuellement: } \\
\text { Capable }\end{array}$ & $\begin{array}{l}\text { Debilitant, Amollissar } \\
\text { Affaiblissant. }\end{array}$ \\
\hline $\begin{array}{l}\text { Capable } \\
\text { Excellent }\end{array}$ & fortification \\
\hline Doué & Abri (armé) \\
\hline Calé (fam.) & Bastide \\
\hline Ferré (fam.) & Bastion \\
\hline Expérimenté & Blockhaus \\
\hline Habile & Casemate \\
\hline Talentueux & Camp retranché \\
\hline Intelligent & Citadelle \\
\hline Adroit & Travaux militaires \\
\hline Adapté & Fort \\
\hline
\end{tabular}

Annexe 4 : Exemple d'article du dictionnaire de Boussinot 